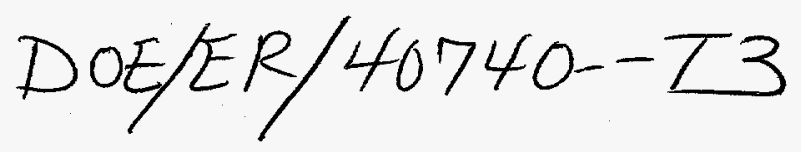

\title{
The Theory of Hadronic Systems
}

\section{DOE Grant No. DE-FG03-92ER40740 New Mexico State University Las Cruces, NM 88003}

\author{
William R. Gibbs
}

March 16, 1995

\section{DISCLAIMER}

\begin{abstract}
This report was prepared as an account of work sponsored by an agency of the United States Government. Neither the United States Government nor any agency thereof, nor any of their employees, makes any warranty, express or implied, or assumes any legal liability or responsibility for the accuracy, completeness, or usefulness of any information, apparatus, product, or process disclosed, or represents that its use would not infringe privately owned rights. Reference herein to any specific commercial product, process, or service by trade name, trademark, manufacturer, or otherwise does not necessarily constitute or imply its endorsement, recommendation, or favoring by the United States Government or any agency thereof. The views and opinions of authors expressed herein do not necessarily state or reflect those of the United States Government or any agency thereof.
\end{abstract}




\section{Contents}

1 Introduction $\quad 3$

2 Research Highlights $\quad 4$

3 Research Summary 6

3.1 Isospin Breaking in the Pion-Nucieon system . . . . . . . 6

3.2 Subthreshold Amplitudes in the $\pi \mathrm{N}$ System . . . . . . . . 12

3.3 Neutron-Proton Charge-Exchange . . . . . . . . . . . . 13

3.4 Transparency in Pion Production . . . . . . . . . . 17

3.5 Energy Dependence of Pion DCX . . . . . . . . . . . . 17

3.6 Direct Capture of Pions into Deeply Bound Atomic States . 20

3.7 Knock Out of Secondary Components in the Nucleus . . . . . 21

3.8 Radii of Neutron Distributions in Nuclei . . . . . . . . . . . . . 22

3.9 The Hadronic Double Scattering Operator . . . . . . . . . . . 24

3.10 Pion Scattering and Charge Exchange from Polarized Nuclei 24

3.11 Pion Absorption in Nuclei . . . . . . . . . . . . . . 25

3.12 Modification of of Nucleon Structure in Nuclei . . . . . . . 28

3.13 Antiproton Annihilation in Nuclei . . . . . . . . . 28

4 Proposed Research 29

5 Personnel $\quad 32$

References $\quad 33$

Budget 39

$\begin{array}{ll}\text { Assurances } & 42\end{array}$ 


\section{List of Figures}

3.1 An Example of Fits to Elastic Scattering Data . . . . . . . . . 7

3.2 Comparison of the s-wave Amplitudes . . . . . . . . . . 8

3.3 Comparison of the p-wave Spin-independent Amplitudes . . . . . . 9

3.4 Comparison of the p-wave Spin-depentent Amplitudes . . . . . . 10

3.5 Forward Amplitudes Near the Charge-exchange Minimum . . . . . 11

3.6 S-wave Contributions to the Sigma Term . . . . . . . . . . . 13

3.7 Energy Dependence of $n \mathrm{p}$ Charge Exchange at $\mathrm{Q}=0 \ldots \ldots \ldots$

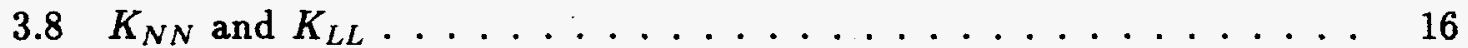

3.9 Pion Double Charge Exchange on ${ }^{58} \mathrm{Ni} \ldots \ldots \ldots \ldots \ldots$

3.10 Valence Neutron Densities . . . . . . . . . . . . . . . 23

3.11 Asymmetry and Cross Sections for ${ }^{13} \mathrm{C} \ldots \ldots \ldots \ldots . \ldots 25$

3.12 Asymmetry for Elastic Scattering from ${ }^{15} \mathrm{~N} \ldots \ldots \ldots \ldots$

3.13 Deuteron Spectrum from Pion Absorption on ${ }^{4} \mathrm{He} \ldots \ldots \ldots . . .27$ 


\section{Chapter 1}

\section{Introduction}

This document is a request for a renewal of the grant DE-FG03-92ER40740 from the Department of Energy for work on theoretical research for the study of the hadronic interaction to be carried out at New Mexico State University in the period 1995-1998.

The research activities are in several areas united by the central theme of the study of the hadronic interaction. An underlying motif in all of the work is an attempt to understand the strong interaction in the full sense of the word; not only its fundamental source but the way in which it manifests itself in the systems it binds. These may be nuclei or smaller systems, such as composite mesonic states or the nucleons themselves.

Following is a summary of the work done under this grant during the past three years. 


\section{Chapter 2}

\section{Research Highlights}

This section contains a brief list of res:- - ch accomplishments which are treated in more detail in the following chapter.

- Analysis of low-energy pion-nucleon amplitudes revealed isospin breaking in the $s$-wave amplitudes. The effect is of the size that might be expected but does not fit into any of the current theories in a ready fashion.

- A modification of one pion exchange, based on subnucleonic degrees of freedom, allowed an immediate, and very simple, representation of cross section and spintransfer data in the np charge exchange reaction over a wide range of energies. Without this modification the predicted results bear no resemblance to the data. It appears that pion exchange has a wider range of applicability than previously thought.

- Comparison of a classical simulation to data from inclusive pion scatiering revealed the existence of a îlling of the "dip region" (between quasifree and delta production) similar to that observed in electron scattering.

- It was shown that the cross section for quasi-free knockout could be represented in terms of interfering amplitudes for knock out of the particle detected plus knockout of the rest of the nucleus for the reaction ${ }^{4} \mathrm{He}\left(\pi^{+}, \pi^{+} \mathrm{p}\right)^{3} \mathrm{H}$.

- Comparison of a classical simulation with pion absorption data on ${ }^{4} \mathrm{He}$ showed a number of signals of initial- and final-state interactions. Perhaps the most interesting is the existence of "pick-up" deuterons, clearly seen in the experimental data. 
- It was demonstrated that the resonance-like peak in low-energy pion double charge exchange can be understood without recourse to the assumption of the existence of a dibaryon.

- The neutron radii of the calcium isotopes ${ }^{40} \mathrm{Ca},{ }^{42} \mathrm{Ca},{ }^{44} \mathrm{Ca}$ and ${ }^{48} \mathrm{Ca}$ were determined with an uncertainty of the order of $1-2 \%$. The radius of the valence neutrons in ${ }^{42} \mathrm{Ca}$ was found to be anomalously large.

- It was shown that pion scattering and charge exchange from polarized ${ }^{13} \mathrm{C}$ is largely understood. There remain some problems with the scattering from ${ }^{15} \mathrm{~N}$.

- It was demonstrated that the $\left(\pi^{-}, p\right)$ reaction is a viable technique for forming deeply-bound pionic atoms. 


\section{Chapter 3}

\section{Research Summary}

\subsection{Isospin Breaking in the Fion-Nucleon System}

The study of a classification of isospin breaking in the pion-nucleon system was recently completed and published[1]. To our knowledge no such classification had previously been made in this system. Such a formalism is essential in order to distinguish the different types of isospin breaking and determine which experiments need to be performed in order to completely specify the system. The classification of these effects takes the form of a tensorial ranking in isospin operators with certain ranks (and specific operators) being associated (approximately) with given physical origins. For example, $\rho-\omega$ mixing has a different isospin-breaking character, in the pion-nucleon interaction, than $\pi-\eta$ mixing. The two effects can be separated experimentally (at least in principle).

While the principal task of the above work is to classify the isospin breaking in the pion-nucleon system, we also point out [8] that the assumption of the non-existence of an isospin-breaking interaction of order $\Delta I=3$ provides a relationship among the widths of the different charge states of the $\Delta$ (in contrast to the masses whose relations are well known) which apparently has not been noticed before.

In current work along the same lines we have made a study analyzing the experimental evidence for isospin breaking in the $\pi N$ system. Most evidence for such breaking is negative in the measurements which have been made to date, except for pion-deuteron scattering and our test of the triangle rule described below.

The total cross section measurements made by Pedroni et al. [14] on deuterium, using positive and negative pion beams, show a clear difference in the two cross sec- 


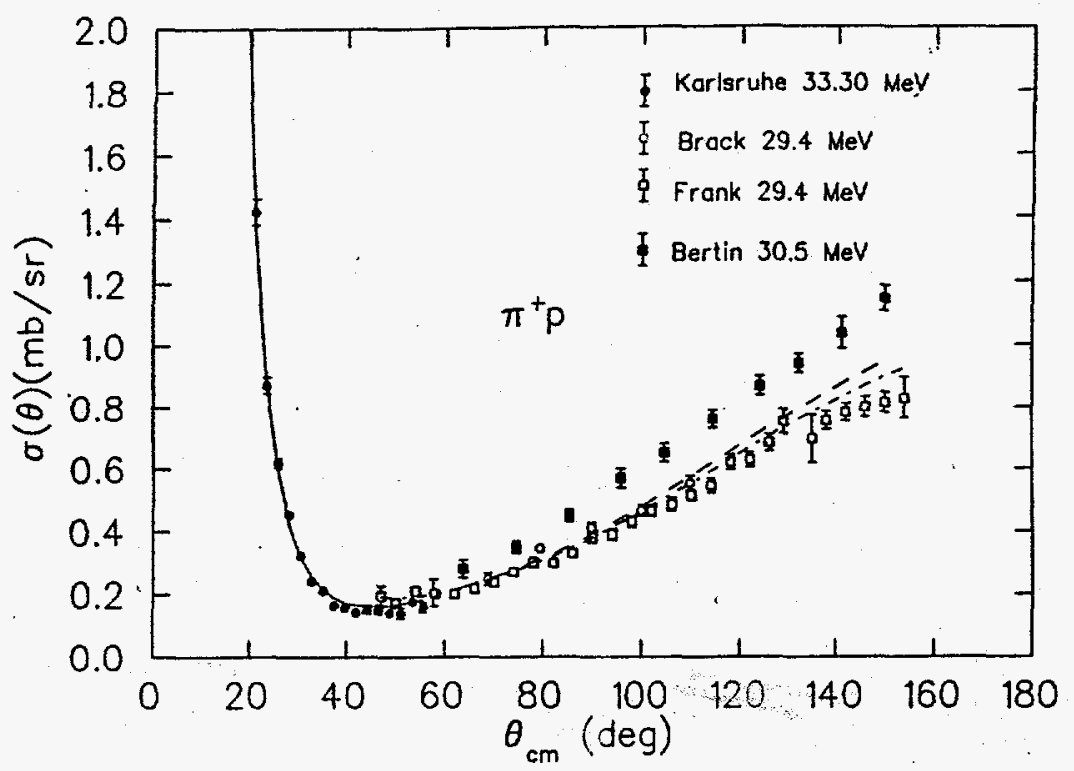

Figure 3.1: Fit to the $\pi^{+} \mathrm{p}$ data at $30 \mathrm{MeV}$.

tions. This difference has traditionally been interpreted as due the mass differences in the four charge states of the $\Delta$ resonance which are, in turn, thought to arise from the mass differences of the up and down quarks and the Coulomb interaction. We see no reason to question this interpretation. There also exist measurements of the differential cross section for pion scattering from deuterium (made at TRIUMF, LAMPF and Saturne) which give information on isospin breaking. While interpretations given in the paper by G. Smith et al. [15] on the published measurements at TRIUMF appear to disagree with the results of Pedroni, we find a very reasonable agreement between the two sets of measurements.

While $\pi$-d data address only one of the six possible isospin breaking amplitudes, a test of $\rho-\omega$ mixing is none the less possible since this source of isospin breaking contributes to this amplitude. We find that the new [16] low-energy data on the charge asymmetry in pion-deuteron scattering are not completely consistent with the effects of the Coulomb interaction and quark mass differences only. The addition of charge-symmetry breaking due to $\rho-\omega$ mixing, using the same parameters needed to explain the isospin breaking observed in the nucleon-nucleon system[17], results in a larger discrepancy between theory and data. A natural first interpretation of this result is that $\rho-\omega$ mixing is not the major cause of isospin breaking in the nucleonnucleon system, as has generally been believed. If the effect were of the opposite sign 


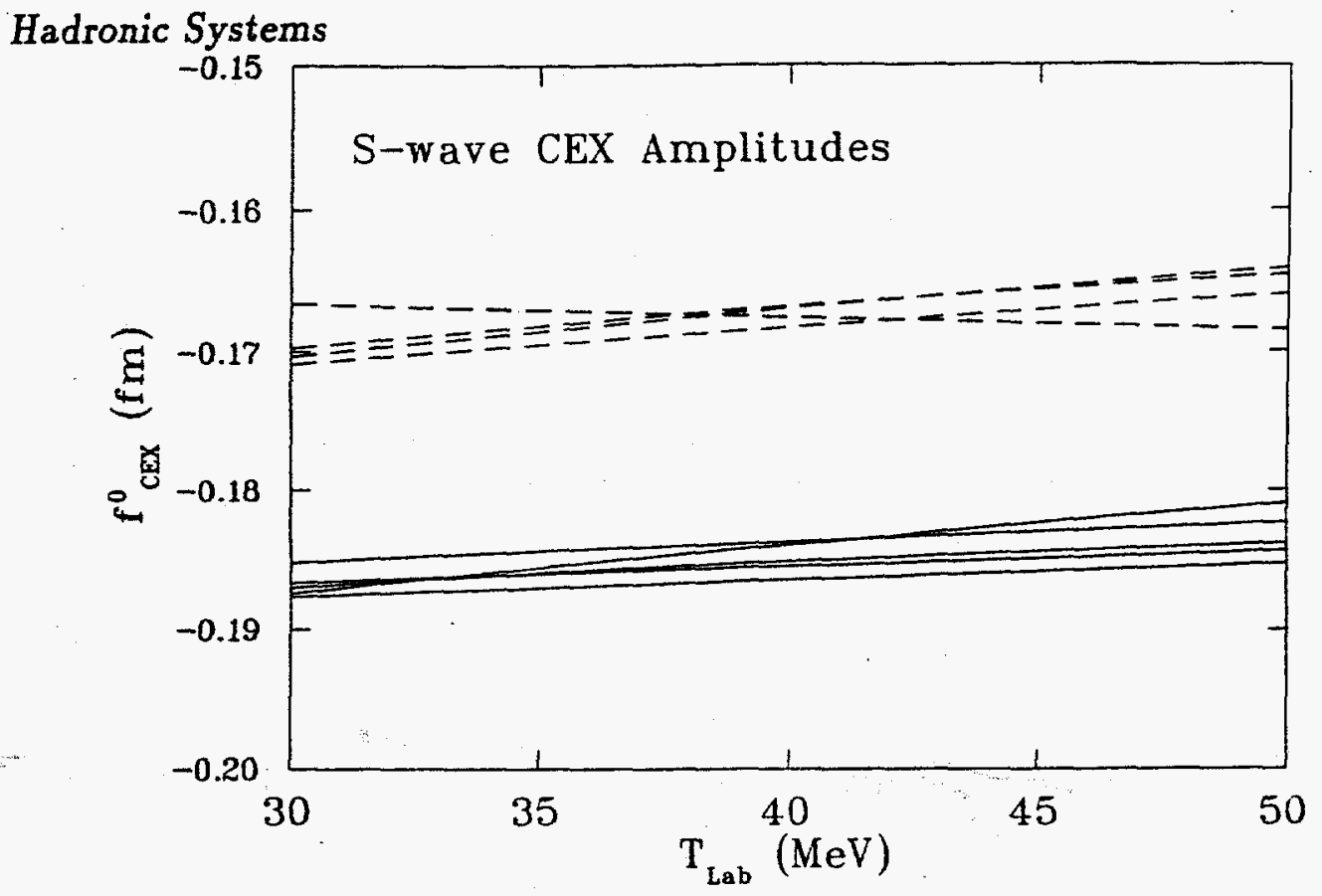

Figure 3.2: Comparison of the s-wave Amplitudes

the agreement would be considerably improved.

The direct analysis of low-energy $\pi$-nucleon data for isospin breaking has recently been completed and a Letter describing this work is in press. Recent measurements of pion-nucleon scattering have provided data of exceptionally high quality below and around $50 \mathrm{MeV}$. We have used potentials models (three local and two separable) which give excellent fits to data to provide representations of the amplitudes for elastic scattering. The data fit are in the region $30-50 \mathrm{MeV}$ and the energy dependence of the amplitudes is very simple ( $s$-wave nearly constant and p-wave nearly linear with energy). In addition, in this energy region the spin-dependent amplitude plays a small role and the imaginary part of the amplitude is small (typically of the order of $10 \%$ of the real part) so that ambiguities are essentially non-existent in this energy region. The reason that potential models are used (whereas a polynomial in center-of-mass momentum would have done as well for energy dependence) is to include the Coulomb and mass-splitting effects via a coupled channel model [7].

Figure 3.1 shows a typical fit to these data. It is observed (in this figure and in the rest of the work) that the "new" data [18] are consistent with each other and inconsistent with the older data of Bertin et al. [19]. The amplitudes can be determined in this manner to an accuracy of the order of $1 \%$. From these results the charge exchange cross section can be predicted by the isospin conserving relation 


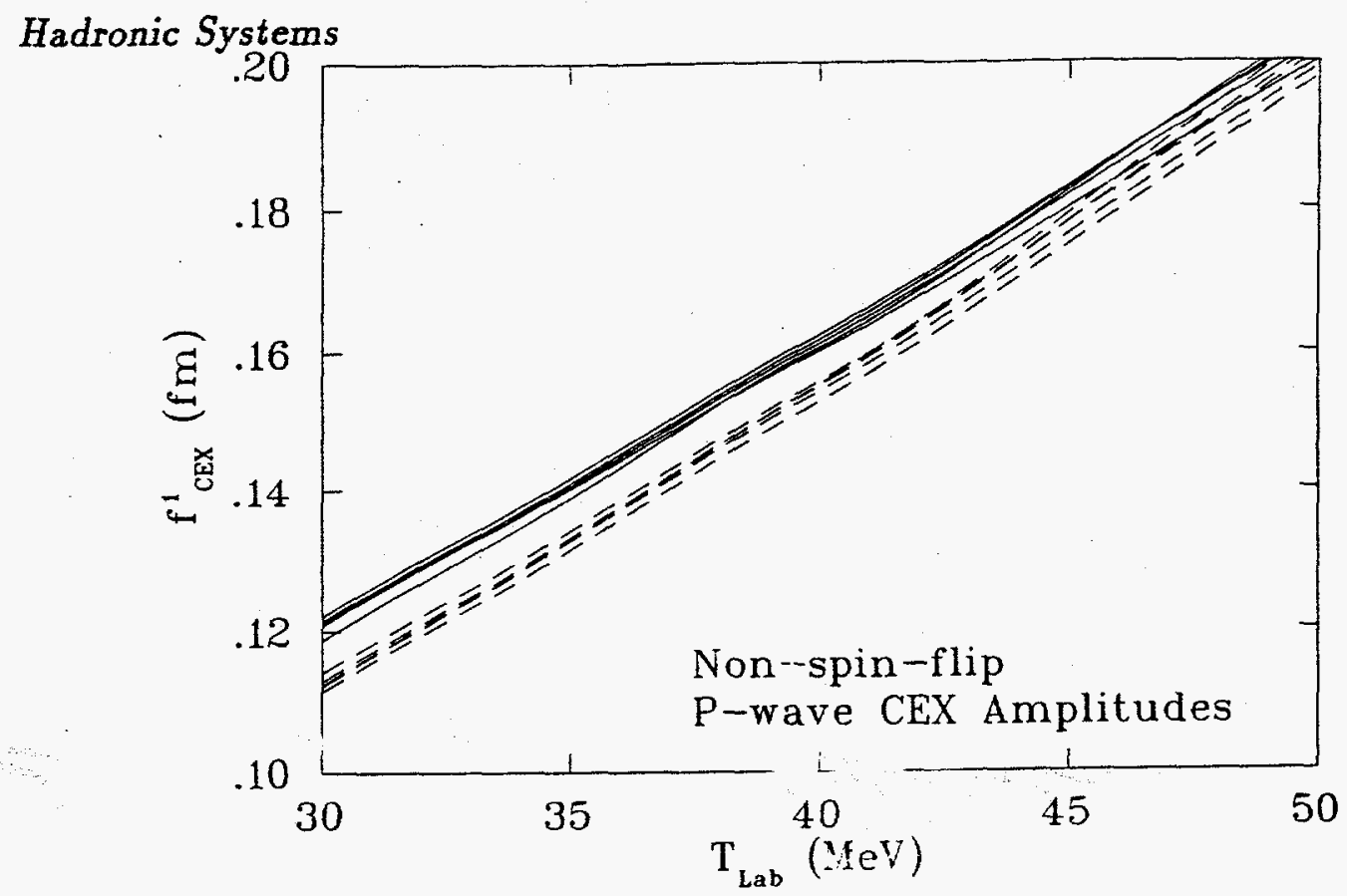

Figure 3.3: Comparison of the p-wave Spin-independent Amplitudes

$$
\sqrt{2} f_{c e x}=f_{\pi^{+}}-f_{\pi^{-}}
$$

and, in particular, the position of the deep minimum at zero degrees near $44 \mathrm{MeV}$ can be accurately determined. The existence of this minimum in the charge exchange cross section at zero degrees is of crucial importance since its position can be determined much more precisely experimentally than the absolute magnitude of the charge exchange cross section.

These predictions have been compared with the charge exchange amplitudes themselves and directly with the data[20]. We find that there is a significant discrepancy. Three separate quantities can be compared between the two sets of data: the s-wave amplitudes and the two p-wave amplitudes, spin independent and spin dependent. The comparison of the s-wave amplitudes is shown in Figure 3.2. The predictions are shown as the dotted curves and the direct fits to the charge exchange data are shown as solid lines. It is seen that the two groups are well separated. The breaking in this wave is seen to be approximately constant and of the order of $7 \%$.

The comparison of the spin-independent $p$-wave amplitudes is shown in Figure 3.3. It is seen that at low energy there is an apparent breaking of the opposite sign as that seen in the case of the $s$ waves. The spin-dependent amplitudes are shown in Figure 3.4. Here there is no evidence for breaking but the amplitude is smaller and ill 
determined by the present data. Polarization data have recently been taken for the $\pi^{+} p$ elastic scattering (but are not yet available) and there is a planed experiment for charge exchange polarization to be run this summer. These additional data may change the situation with regard to the spin-dependent amplitude.

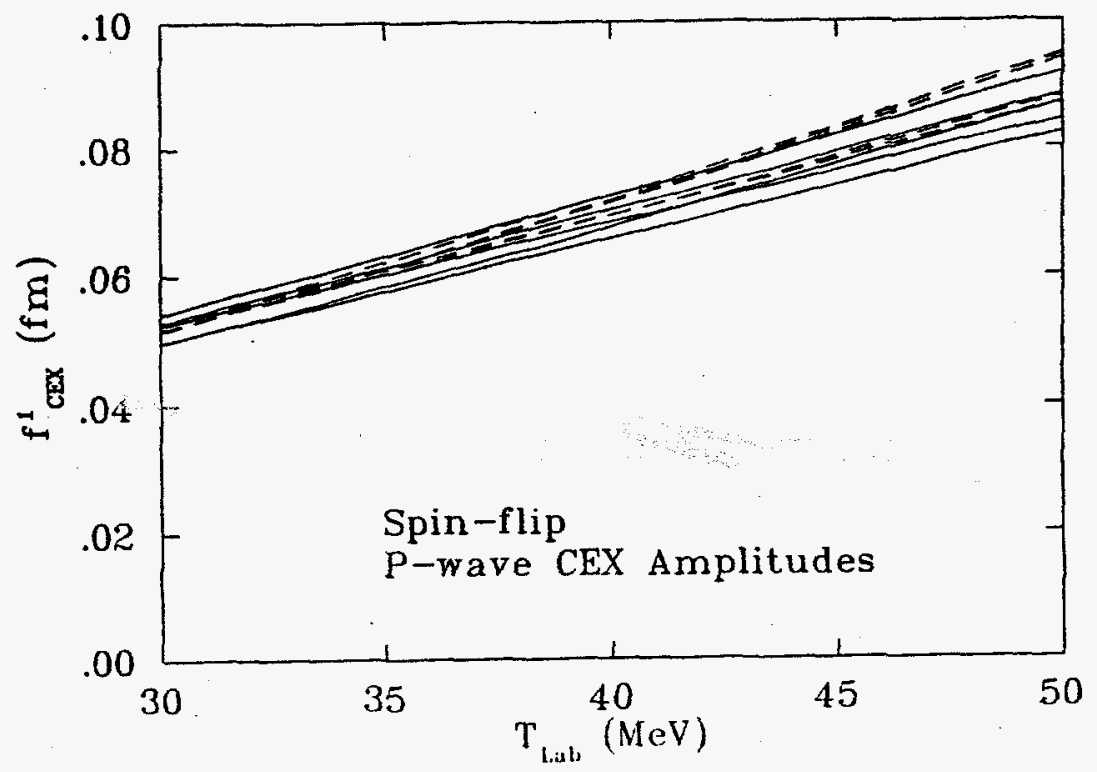

Figure 3.4: Comparison of the p-wave Spin-dependent Amplitudes

Of course one cannot tell from the present work where the breaking occurs in Eq. 3.1 ; it could as well be in the charge exchange or the $\pi^{+} / \pi^{-}$scattering (or a combination of the two). The meson contributions to the isospin breaking amplitude are $\pi \eta$ mixing in charge exchange and $\rho \omega$ mixing in scattering. If the entire breaking is assumed to have the form of $\rho \omega$ mixing (the $a_{3}$ amplitude in our classification) the approximate size and sign are correct to explain the breaking seen in low energy $\pi$-deuteron scattering. In order to separate the two effects in a more reliable way, an additional experiment needs to be done, perhaps a comparison of ${ }^{3} \mathrm{He}\left(\pi^{-}, \pi^{0}\right)^{3} \mathrm{H}$ with ${ }^{3} \mathrm{H}\left(\pi^{+}, \pi^{0}\right)^{3} \mathrm{He}$ at zero degrees in the region of the charge-exchange minimum at low energies.

It is worthwhile to note that $\rho \omega$ mixing parameters can be taken directly from the nucleon-nucleon sector [17] assuming universality of the $\rho$ coupling constant. We have done this and our results agree with Ref. [21]. The prediction is of the right magnitude but the wrong sign to explain the breaking seen in the triangle rule. There is considerable controversy over the $\rho \omega$ charge symmetry breaking potential [22]. 


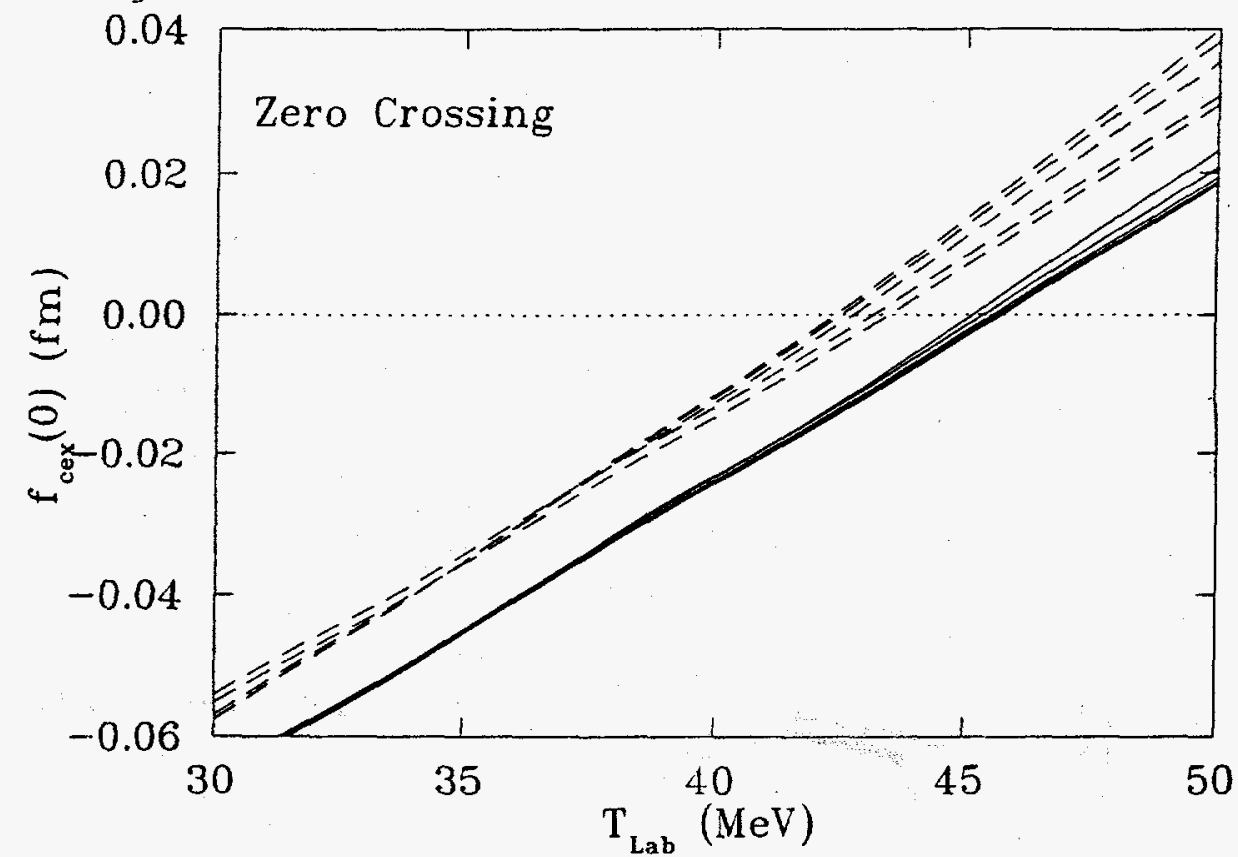

Figure 3.5: Real part of the forward amplitudes near the charge-exchange minimum

One can try to make a direct comparison with the prediction from quark masses. Following the work of Weinberg [23], the charge symmetry breaking in neutral pion scattering from neutrons and protons can be estimated. While such an experiment is not currently feasible, the amplitudes for this process can be rewritten in terms of amplitudes which are measurable.

$$
A_{\pi^{0} n}-A_{\pi^{0} p}=\frac{m}{4 \pi(m+\mu) F_{\pi}^{2}}\left(m_{d}-m_{u}\right)=-\sqrt{\frac{2}{3}} a_{2}-2 \sqrt{\frac{2}{15}} a_{7}+\frac{2}{\sqrt{5}} a_{8}
$$

By assuming the the coefficient $a_{2}$ can be neglected in this expression and (following Weinberg) $m_{d}=7.5 \mathrm{MeV}$ and $m_{u}=4.2 \mathrm{MeV}$, we find $a_{7}=-0.007 \mathrm{fm}$. which contributes $0.0025 \mathrm{fm}$. to the $0.013 \mathrm{fm}$. breaking observed in the s-wave. The coefficients in this expression are uncertain by as much as a factor of 2 so low-energy chiral lagrangians might supply as much as one half of the breaking seen. It is not clear that the neglect of $a_{2}$ in the above expression is justified.

This work was done in collaboration with $\mathrm{Li} \mathrm{Ai}$ and W. B. Kaufmann. 


\subsection{Subthreshold Amplitudes in the $\pi \mathrm{N}$ System}

The amplitudes obtained from the potentials in the previous sections are defined in regions other than that in which the scattering data were taken. In particular they can be calculated in the subthreshold region. This region is of interest because both the pion-nucleon pole and the Cheng-Dashen (CD) point lie there. We intend to treat the pion-nucleon pole in the near future in order to attempt to obtain the pionnucleon coupling constant from the residue but for the moment we have only begun the study of the Sigma term and present here preliminary results.

The Cheng-Dashen point is located in the two dimensional Mandelstam plane with only on-shell values needed. Its position can be specified in several equivalent ways. We will take here $s=u$ and $t=2 \mu^{2}$. In terms of the center-of-mass pion momentum $\omega$,

$$
\omega_{C D}=\frac{\mu^{2}}{2 m} \approx 0.05 \mathrm{fm} .
$$

As was pointed out by Ericson[24], the value of the cosine of the scattering angle at the Cheng-Dashen point is $\approx \frac{1}{200}$ so the p-wave is of little importance for the $\Sigma$-term. Thus we look only at the s-wave contribution.

In this case we have the two amplitudes, $S_{\frac{3}{2}}$ and $S_{\frac{1}{2}}$, corresponding to isospin $3 / 2$ and $1 / 2$ respectively. Note that the isovector amplitude vanishes at the CD point so that (still neglecting the p-wave at this point and assuming good isospin for the moment) these two amplitudes must be equal. Thus, in principle, one needs only an extrapolation of the s-wave $\pi^{+}$p scattering amplitude to obtain $\Sigma$. However it is an important check on the fits and the entire theory to see that the two amplitudes do indeed cross at this point. Of course the question of isospin breaking enters again.

The fits done in the previous section were made with the "truncated" KleinGordon equation, i.e. the term quadratic in the potential was dropped as is common in constructing and solving pion-nucleon and pion-nucleus potentials. This is normally justified on the basis that $\omega$ is large compared with the potential. However, if we wish to extrapolate to a point where $\omega$ is nearly zero this is clearly a poor approximation. In fact if the quadratic term is neglected (since the remaining potential is then linear in $\omega$ ) the two amplitudes will cross at $\omega=0$ with values zero. Hence the value of $\Sigma$ obtained from any analysis with the truncated Klein-Gordon equation is destined to be near zero. For this reason the data is being refit with the quadratic term included. Figure 3.6 shows a preliminary result for the two amplitudes (with the normalization appropriate to make them represent $\Sigma$ in $\mathrm{MeV}$ ). 
Hadronic Systems

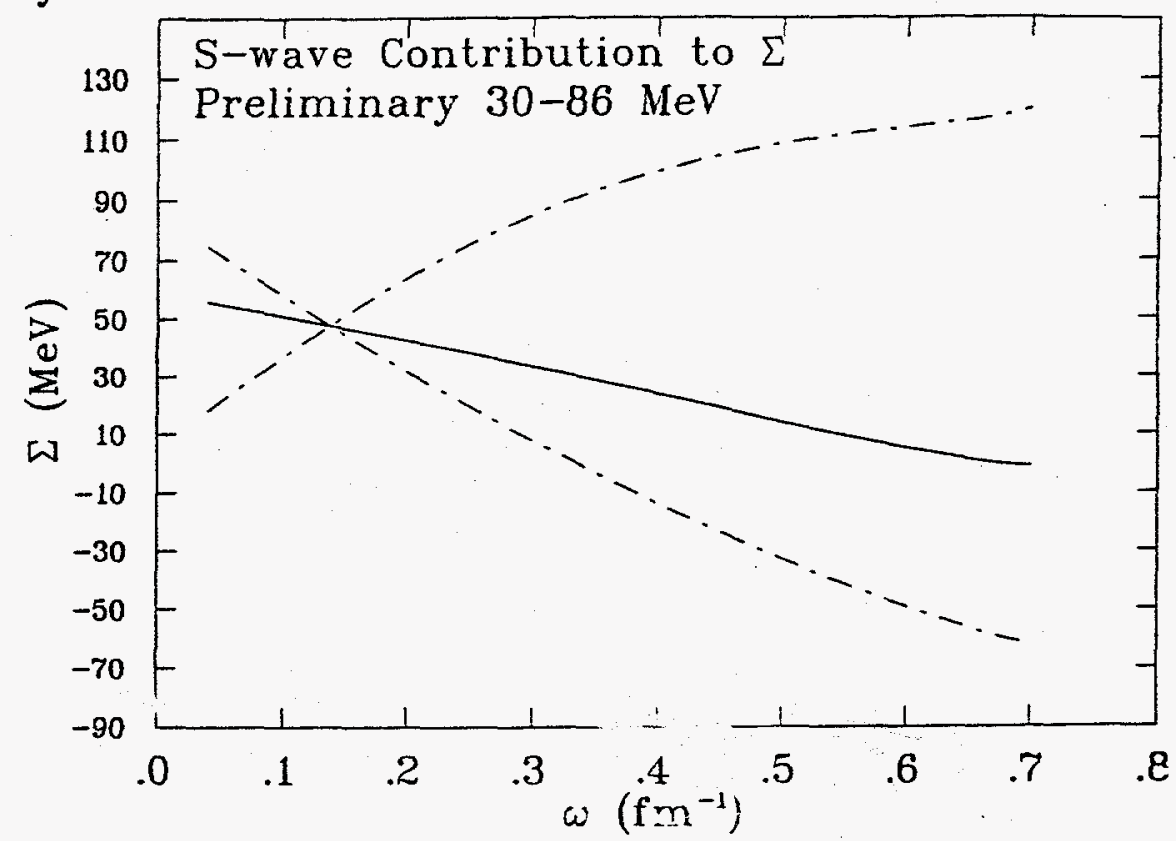

Figure 3.6: S-wave contributions to the Sigma term as a function of the center-of-mass pion energy. Note the crossing of the two isospin contributions near the Cheng-Dashen point at $\omega=0.05 \mathrm{fm}$.

\subsection{Neutron-Proton Charge-Exchange}

Neutron-proton charge exchange at zero degrees (i.e. np scattering at 180 degrees) shows a sharp peak in the differential cross section. This is precisely where the longrange exchange of a charged pion should dominate, but one pion exchange gives zero cross section at $q=0$. Investigation of the one-pion-exchange amplitude shows that the $\ell=0$ component is large (non-unitary in fact) and negative while all other contributions are positive. Removing the s-wave component leads to a cross section of about the right size. A number of authors have considered various ways of suppressing the short range contribution to the amplitude but each of them has some problem (usually they a-e energy dependent). We noticed that the removal of the delta function in the one-pion-exchange potential can lead to a removal of the unwanted s-wave contribution if it is done in the context of pion exchange between the quarks which make up the nucleon. We recently found that this same procedure gives a good qualitative representation of the spin-transfer properties of the $n-p$ charge exchange reaction when compared with recent data [25]. We then added short-range potentials based on two- and three-pion exchange to obtain quantitative agreement with the 
data. A brief summary of the basic argument and result follows.

Since the s-wave component of the one-pion-exchange amplitude is given by

$$
\frac{1}{4 \pi} \int d \Omega \frac{\sigma_{1} \cdot \mathbf{q} \sigma_{2} \cdot \mathbf{q}}{q^{2}+\mu^{2}}=\frac{1}{3} \frac{q^{2} \sigma_{1} \cdot \sigma_{2}}{q^{2}+\mu^{2}}
$$

it has a large-q limit of unity leading to a $\delta$-function in the potential

$$
V_{0}(r) \propto \mu^{2} \frac{e^{-\mu\left|\mathbf{r}_{1}-\mathbf{r}_{2}\right|}}{\left|\mathbf{r}_{1}-\mathbf{r}_{2}\right|}+\delta\left(\mathbf{r}_{1}-\mathbf{r}_{2}\right)
$$

For separations of the quarks which are less than the size of the pion we need to cut off the potential, perhaps with a form like;

$$
V(r)=V_{0}(r)\left(1---(R)^{2}\right) .
$$

Transforming back into momentum space and taking the limit of $R \rightarrow 0$ the modified amplitude becomes

$$
\frac{\sigma_{1} \cdot \mathbf{q} \sigma_{2} \cdot \mathbf{q}}{q^{2}+\mu^{2}} \rightarrow \frac{\sigma_{1} \cdot \mathbf{q} \sigma_{2} \cdot \mathbf{q}}{q^{2}+\mu^{2}}-\frac{1}{3} \sigma_{1} \cdot \sigma_{2}=\frac{1}{3} \frac{q^{2} S_{12}}{q^{2}+\mu^{2}}-\frac{1}{3} \frac{\sigma_{1} \cdot \sigma_{2} \mu^{2}}{q^{2}+\mu^{2}}
$$

where $S_{12} \equiv 3 \sigma_{1} \cdot \hat{\mathbf{q}} \sigma_{2} \cdot \hat{\mathbf{q}}-\sigma_{1} \cdot \sigma_{2}$. We see that only the spin-spin part of the amplitude (and potential) is modified.

For charged pion exchange only, the cross section is given by

$$
\sigma(q)=\frac{4}{3} N^{2} g^{2}(q)\left[1+3 f^{2}(q)-2 f(q)\right] ; \quad f(q) \equiv \frac{q^{2}}{q^{2}+\mu^{2}} ; \quad g(q) \equiv\left(\frac{\Lambda^{2}}{q^{2}+\Lambda^{2}}\right)^{2}
$$

The momentum transfer in these relations is given by $q^{2}=2 k^{2}(1+\cos \theta)$, and the normalization of the cross section by:

$$
N=\left(\frac{M_{n}}{m_{\pi}}\right)^{2} \frac{1}{\sqrt{s}} \frac{f_{\pi}^{2}}{4 \pi}\left(\frac{\Lambda^{2}-\mu^{2}}{\Lambda^{2}}\right)^{2} .
$$

Here $s$ is the square of the center-of-mass energy of the two nucleons. The factor of $\frac{1}{\sqrt{3}}$ provides a scaling factor which is observed to be consistent with the data over a range of beam energies from $200 \mathrm{MeV}$ to $60 \mathrm{GeV}$ (see Figure 3.7). 


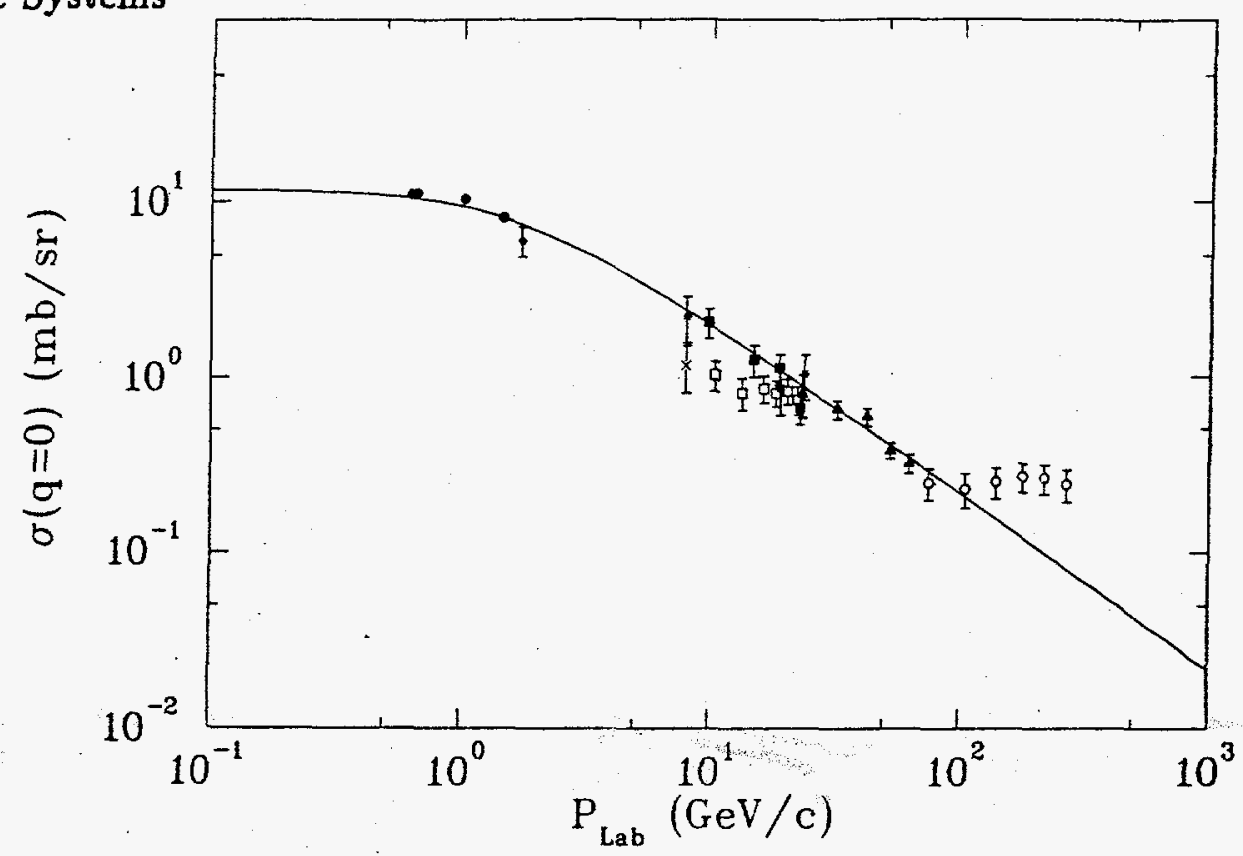

Figure 3.7: Energy dependence of $\mathrm{np}$ charge exchange at $\mathrm{Q}=0$. The solid line is obtained by scaling the $800 \mathrm{MeV}$ data by a factor of $1 / \mathrm{s}$.

The spin transfer observables are given by:

$K_{S S}(q)=\frac{-\frac{1}{3}+3 f^{2}(q)-2 f(q)}{1+3 f^{2}(q)-2 f(q)} ; K_{L L}(q)=\frac{-\frac{1}{3}-3 f^{2}(q)+2 f(q)}{1+3 f^{2}(q)-2 f(q)} ; K_{N N}(q)=K_{L L}(q)$.

If the $\delta$-function were not removed the values of the spir observables would be:

$$
K_{S S} \equiv+1 ; \quad K_{L L} \equiv-1 ; \quad K_{N N} \equiv-1
$$

For the inclusion of both the charged and neutral one pion exchange the expressions for the amplitudes are only slightly more complicated. Comparison of these predictions with $K_{N N}$ and $K_{L L}$ is shown in Figure 3.3. The dashed curve shows the prediction from charged pion exchange alone while the two solid curves include the exchange of the neutral pion as well. Note that the data are largely independent of the beam energy as predicted.

The spin-spin component of the modified potential is given by

$$
V_{s s}(r)=\frac{f^{2}}{4 \pi}\left[\frac{e^{-\mu r}-e^{-\Lambda r}}{r}-\frac{\Lambda^{2}-\mu^{2}}{2 \Lambda} e^{-\Lambda r}\right] .
$$




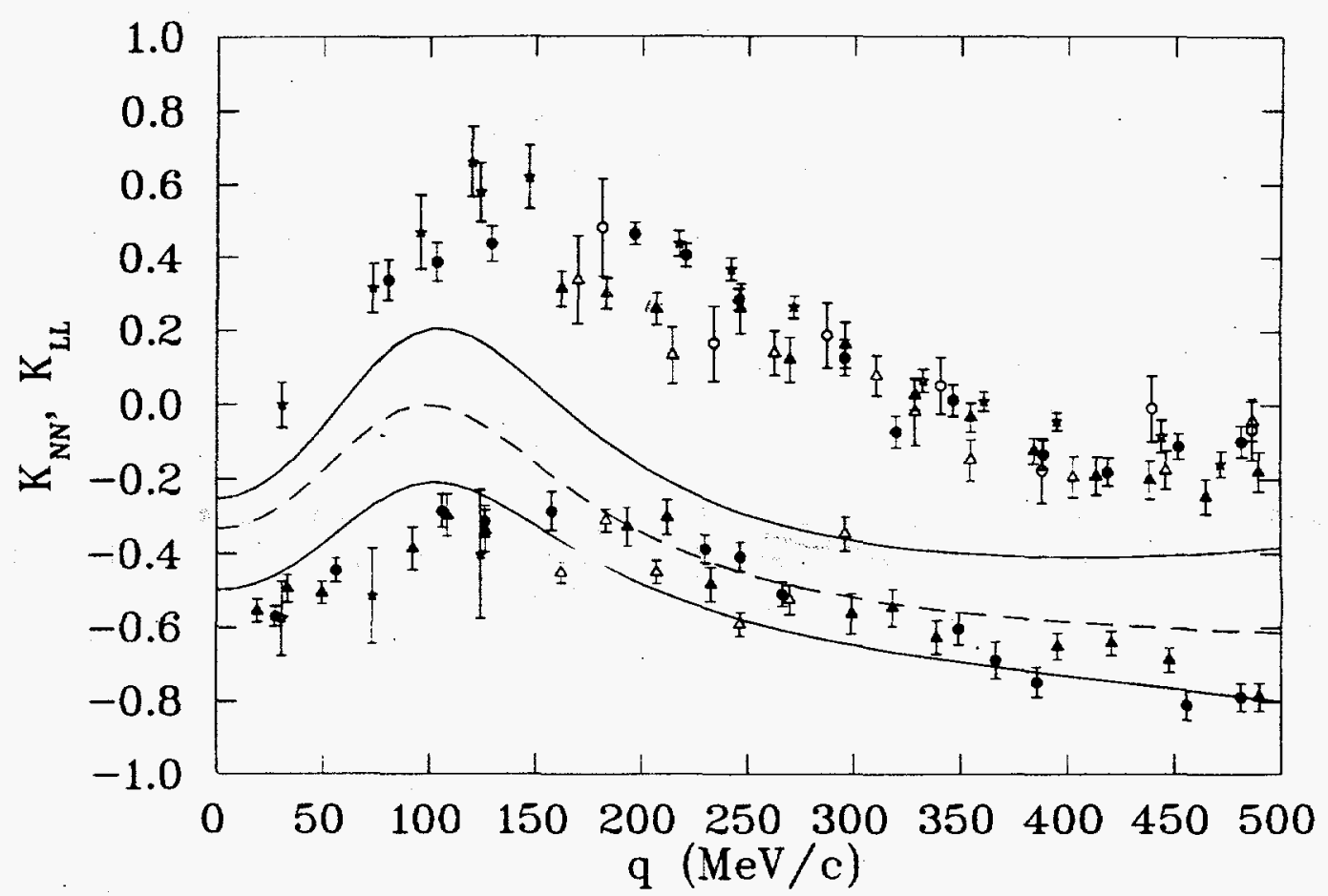

Figure 3.8: $K_{V N}$ (bottom) and $K_{L L}$ (top) as a function of momentum transfer. The data[25] include energies from $485 \mathrm{MeV}$ to $788 \mathrm{MeV}$.

The potential with the $\delta$-function (the original one-pion-exchange distributed over the quark density in the nucleon, or simply multiplied by a form factor for whatever reason) is given by

$$
V_{s s}^{\delta}(r)=\frac{f^{2}}{4 \pi}\left[\frac{e^{-\mu r}-e^{-\Lambda r}}{r}-\frac{\Lambda\left(\Lambda^{2}-\mu^{2}\right)}{2 \mu^{2}} e^{-\Lambda r}\right]
$$

Thus the large difference seen in the cross section and spin-transfer observables is not at all obvious in the form of the potential. The difference is a little clearer when one observes that the coefficient of last term has the proper normalization for a $\delta$-function in the limit of large $\Lambda$ in the second case and not in the first.

This work is being done in collaboration with B. Loiseau. 


\subsection{Transparency in Pion Production}

The study of reactions initiated by pion beams of the order of $500 \mathrm{MeV}$ kinetic energy leads to some very interesting results. This is the energy at which pion production begins to become important. In fact, the low energy portion of the pion spectrum resulting from such beams is dominated by pions that were produced rather than those which underwent large-energy-loss scatterings. These produced pions have an average energy of about $180 \mathrm{MeV}$ or very near the $\Delta_{33}$ energy. Since the mean free path of the $500 \mathrm{MeV}$ incident energy pions is long, the produced pions are created well within the nuclear volume which means that they must traverse a significant amount of nuclear material to escape. Thus, in the spectrum of pions produced, one expects to see a minimum in the region of the 33 resonance corresponding to the fact trat pions produced in thict energy region are either ra-absorbed or scattered to lower energies. In fact no such minimum is observed in we uata. Comparisons with a classical simulation reveal a difference of an order of magnitude in this energy region. Attempts to eliminate this large discrepancy with corrections to the production mechanism or with correlations among initial nucleons have little effect.

The only way that has been found to obtain reasonable agreement with the data is to allow essentially all produced pions to escape the nucleus without interaction. Eliminating the second interactions of all pions leads to a very poor agreement with the data; it seems to be only the produced pions which escape the nucleus.

This work has been published in Physical Review Letters[6].

\subsection{Energy Dependence of Pion DCX}

A resonant-like structure in low-energy pion double charge exchange has been known for some time. It manifests itself in a peak around $35-50 \mathrm{MeV}$ pion kinetic energy.

In studies performed on elastic scattering some years ago we pointed out[26] that the cancellation of the s- and p-wave pion-nucleon amplitudes in this energy region leads to a transparency of the nuclear medium. Calculating the effect from a finite volume of this material, it was shown that poles in the S-matrix appear and there are a number of resonances in each pion-nucleus partial wave. These resonances are in the pion-nucleus system and become broader with higher energy. Normally only one or two might be expected to be visible.

Reactions, on the other hand, tend to show up this effect more clearly since a single (or few) partial waves tend to be more important. Our calculations [26] for 
a general monopole transition showed a clear peak in this region. That is not to say that one can always accurately predict positions of the peak and absolute cross sections for reasons to be discussed below.

In spite of a general understanding of this effect in terms of (more or less) standard physics, a suggestion was recently made[27] that this data provided a signal for the existence of a dibaryon. The authors simply allowed themselves to make up the parameters which would fit the data, ignoring the fact that there had to be a peak in this energy region for other reasons.

For this reason we are doing the calculations to show to what degree we do (and do not) understand the data. There are several problems once one is constrained to do a realistic calculation.

First, the reaction mechanism itself. From the work that we did on the double scattering operator[5] and the one pion exchange interaction in the neutron-proton charge-exchange reaction (see above) it can be scen that the $\delta$-function needs to be removed from the DCX operator. This effect has never been investigated by any group (including our own) to my knowledge. This project is currently under way. A rapid calculation with plane waves (very crude for what we want to look at) shows that a considerable renormalization could result.

Second, the nuclear wave function. Since the relative distance between the two nucleons plays a strong role (that is, in fact, a large part of the interest in the reaction) small changes in the wave function can produce significantly different weightings of the pion-nucleus partial waves and hence change the weighting of the resonances. One might expect that, for certain cases, two or more of the resonances could be seen.

Third, the propagation of the intermediate $\pi^{0}$. This uncertainty has two aspects; the optical model to describe the motion of the pion and the question of which excited states play a role. If one uses closure to sum over the intermediate states (as we do) then the double spin flip cancels, to a large extent, the non-spin-flip amplitude with the effect of decreasing the sharpness of the peak. The intermediate states corresponding to the double spin flip are the Gamow-Teller states which are claimed have a quenched strength. If this is true then the cancellation will be reduced and the observed resonance seen in DCX will be stronger.

Fourth, the optical model itself. This is the description of the incoming and outgoing waves. In general, we have what we think is a reasonable model of this part of the reaction but improvements can certainly be made. The principal variable in this part of the model is the range of the off-shell $t$-matrix. For technical reasons it is somewhat difficult to calculate with a range larger than about $500 \mathrm{MeV} / \mathrm{c}$. For this reason most of our previous calculations were made with values in this range. 


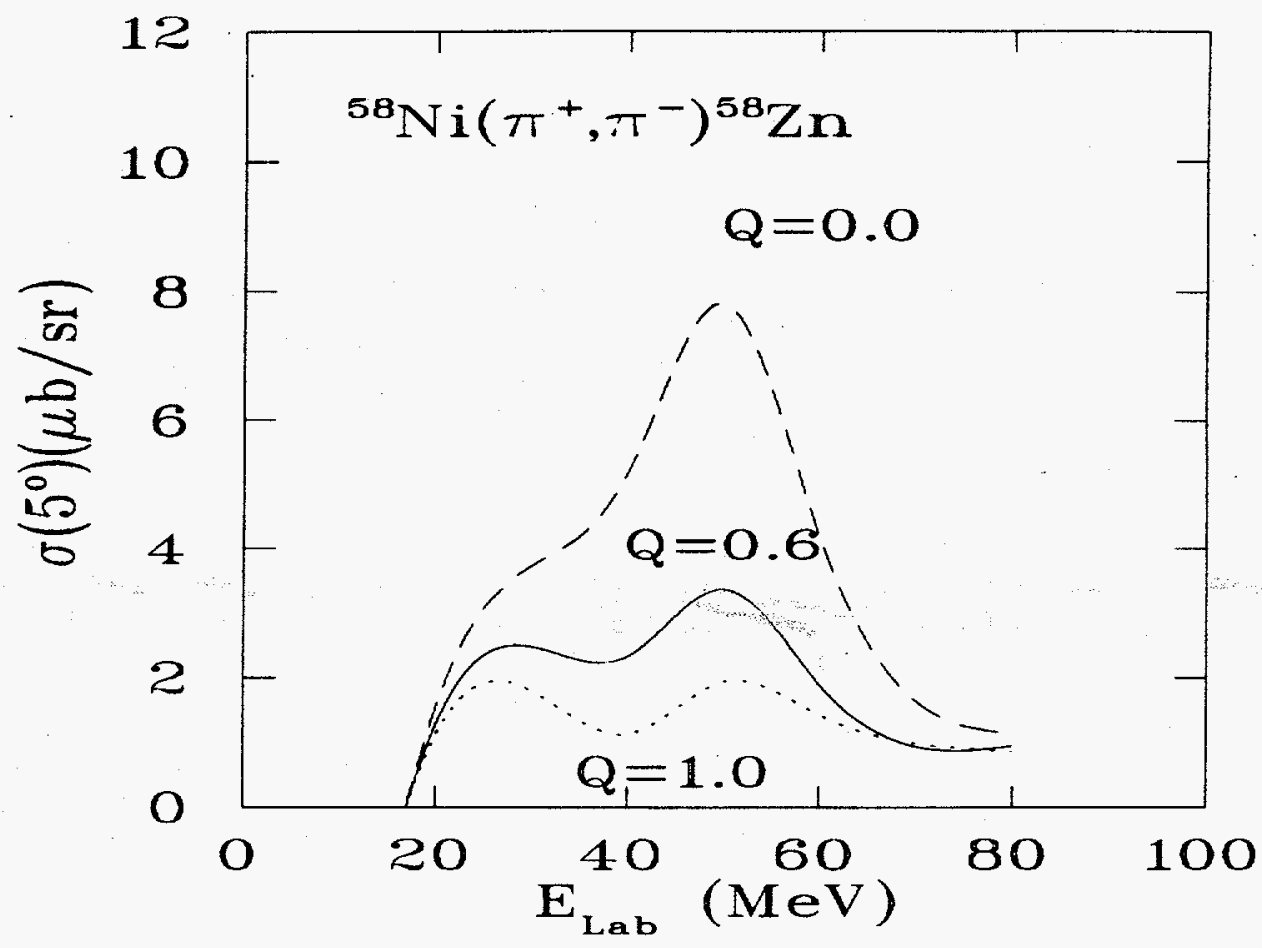

Figure 3.9: Pion double charge exchange on ${ }^{58} \mathrm{Ni}$. The three curves snow the calculation with different quenching factors (Q) applied to the intermediate spin-flip route.

More modern estimates of this quantity put it in the range of $600-800 \mathrm{MeV} / \mathrm{c}$ or even higher.

We have recently performed calculations to see if some of these difficulties can be overcome. For the fourth point we have improved the numerics of the calculation to the point that calculations with an off-shell range of $800 \mathrm{MeV} / \mathrm{c}$ are reasonably accurate $(1 \%)$. For the propagation of the intermediate pion we use the same optical model as for the external legs. For the nuclear wave functions we take what is available and would prefer the simple wave functions if possible. For example, the ground state transitions in ${ }^{44} \mathrm{Ca}$ and ${ }^{48} \mathrm{Ca}$ are taken only from the $f_{7 / 2}$ shell and do not have an "A" amplitude[28] hence are the simplest. For the first problem with the $\delta$-function, 
we continue to calculate with it included for the moment.

Some of our results are summarized in Figure 3.9. In the case of double charge exchange to the analog (and ground) state in ${ }^{58} \mathrm{Ni}$ we see that the resonance peak is very strong indeed if no double spin flip is included. We also show the case of half quenching of the intermediate Gamow-Teller state.

For the ground-state transition in ${ }^{44} \mathrm{Ca}$ the agreement with the data is quite good. The same holds for ${ }^{48} \mathrm{Ca}$. For the analog transitions the peak is clearly present in the data and the calculations but, since the peak in the " $\mathrm{A}$ " amplitude is at a slightly different energy than that in the " $B$ " amplitude (because of the $\delta$-function?) the total peak is shifted.

For ${ }^{18} \mathrm{O}$ the results are particularly interesting. In this case there are two peaks observed experimentally, the second one around $110 \mathrm{MeV}$. It is difficult to see why a second dibaryon would suddenly appear at this point. In our calculation the nuclear wave function is such that a second pean is also seen. The two peaks do not appear at the correct energies however (although they do have about the right spacing).

Our studies of this reaction are continuing but we are reluctant to see evidence for a dibaryon in this peak, even though we are not always able to fit the data perfectly.

This work is being done in collaboration with M. Elghossain, W. B. Kaufmann and N. Auerbach.

\subsection{Direct Capture of Pions into Deeply Bound Atomic States}

The strong interaction of a pion with nuclear material can be studied by means of the measurement of the energy shifts and the non-electromagnetic contributions to the widths of pionic atoms. The effect of the pion-nucleus interaction is stronger the closer the pion is to the nucleus, and hence the higher the atomic number. One advantage for this method of studying scattering is that the pion is in a definite atomic orbital, hence has a single, well-determined, angular momentum with respect to the nucleus. Unfortunately the normal capture mechanism is very inefficient for populating deeply bound states since in the process of the cascade after initial capture in a high orbital state, the pion is more likely to be absorbed by the nucleus than to "descend" to the next orbital.

The lowest s-wave orbital cannot be seen in nuclei much heavier than $\mathrm{Z}=10$. Many possible reactions have been considered for populating these tightiy bound states but 
there is no decisive evidence that any such deeply bound state has been seen. W. B. Kaufmann, P. B. Siegel and I have considered [2] the reaction where an incident negative pion impinges on a nucleus and knocks out a proton from an outer nuclear shell, at the same time being captured in an atomic orbital. While the momentum transfer might seem, at first glance, to be too large to be supported by an atomic orbit, the Fermi momentum of the proton can largely compensate for this seeming default. In fact this larger momentum transfer is often an advantage since many of the shells in heavy nuclei have moderately large angular momenta and thus an appreciable angular momentum (and hence momentum) transfer is necessary to reach a pionic state with small $\ell$. The cross sections are not unreasonably small but the backgrounds are estimated to be large.

\subsection{Knock Out of Secon C...y Components in the Nucleus}

The subject of quasifree knock out is of interest in the study of the hadronic interaction because it allows the possibility of the study of the projectile-nucleon interaction in a nuclear environment. However the degree to which the scattering takes place from a single nucleon (and not, for example, from the other A-1 nucleons in whatever excited state the conglomerate happens to exist) is not clear. The use of pion "quasifree" scattering from a "proton" in ${ }^{4} \mathrm{He}$ offers unique insight into this situation. In this case it is possible to measure the exclusive final state in which the triton is in its ground state. By choosing the charges of the pion, the scattering from the proton or the triton can be selected to be dominant. Indeed studies over a complete angular range indicate that the ratio

$$
\frac{\sigma^{+}(\theta)-\sigma^{-}(\theta)}{\sigma^{+}(\theta)+\sigma^{-}(\theta)},
$$

where the + or - indicates a $\pi^{+}$or $\pi^{-}$beam, shows a large positive excursion in the angular region of quasifree proton scattering and a large negative excursion in the region of quasifree triton knock out. Calculations consisting of the coherent sum of the two knock-out reactions reproduce the qualitative features of the data. The addition of a "shadowing" correction in the form of double scattering improves the agreement but clearly additional physics is needed.

These results, the measurements and some discussion of the missing physics were 
recently published in Physical Review Letters [3]

\subsection{Radii of Neutron Distributions in Nuclei}

The understanding of neutron distributions in nuclei is one of the most important problems in nuclear structure. While electron scattering does an excellent job of determining the charge density, and the proton distribution can be reasonably inferred from these charge distributions, the densities of the (more numerous) neutrons are poorly known. This knowledge is more than just determining the "other half" of the nucleon distributions. Neutrons are the excess particles (the valence particles in many pictures) and in the case of the calcium isotopes studied here there can be zero to eight neutrons outside of a closed shell.

In this case the degree to which nucleons sist tact with each other, rather than the central core, can be examined. Such studies are at the heart of the usual approximations used in calculating nuclear structure. In a recently published analysis [4] J.-P. Dedonder and I applied pion multiple scattering theory to the analysis of pion scattering data by Boyer et al.[29] to make a determination of the absolute total neutron radii to an accuracy of the order of $1 \%$, an error about a factor of 3 smaller than any previous determination.

The valence neutrons in ${ }^{42} \mathrm{Ca}$ are found to have a radius significantly larger than those of ${ }^{44} \mathrm{Ca}$ which are, in turn, somewhat larger than those of ${ }^{48} \mathrm{Ca}$. The densities of the valence neutrons are shown in Figure 3.10.

One interpretation of this result is that since the two neutrons in ${ }^{42} \mathrm{Ca}$ are highly correlated with each other, due to the necessity of coupling the two $f_{\frac{\pi}{2}}$ neutrons to spin zero, they interact strongly and a larger fraction of the binding energy of the system of the last two neutrons is due to the neutron-neutron interaction and less due to the neutron-core interaction than in the case of the other isotopes which have a progressively smaller correlation among the neutrons. For ${ }^{48} \mathrm{Ca}$, since the wave function is represented by a Slater determinant, the neutrons are highly anticorrelated, i.e. as far away from each other as possible, leading to a stronger interaction with the core and a smaller radius. This observation provides a partial understanding of why the proton radius of ${ }^{48} \mathrm{Ca}$ is observed to be about the same size as the proton radius of ${ }^{40} \mathrm{Ca}$. The proton radius does not grow throughout the shell because the valence neutron radius is shrinking. 


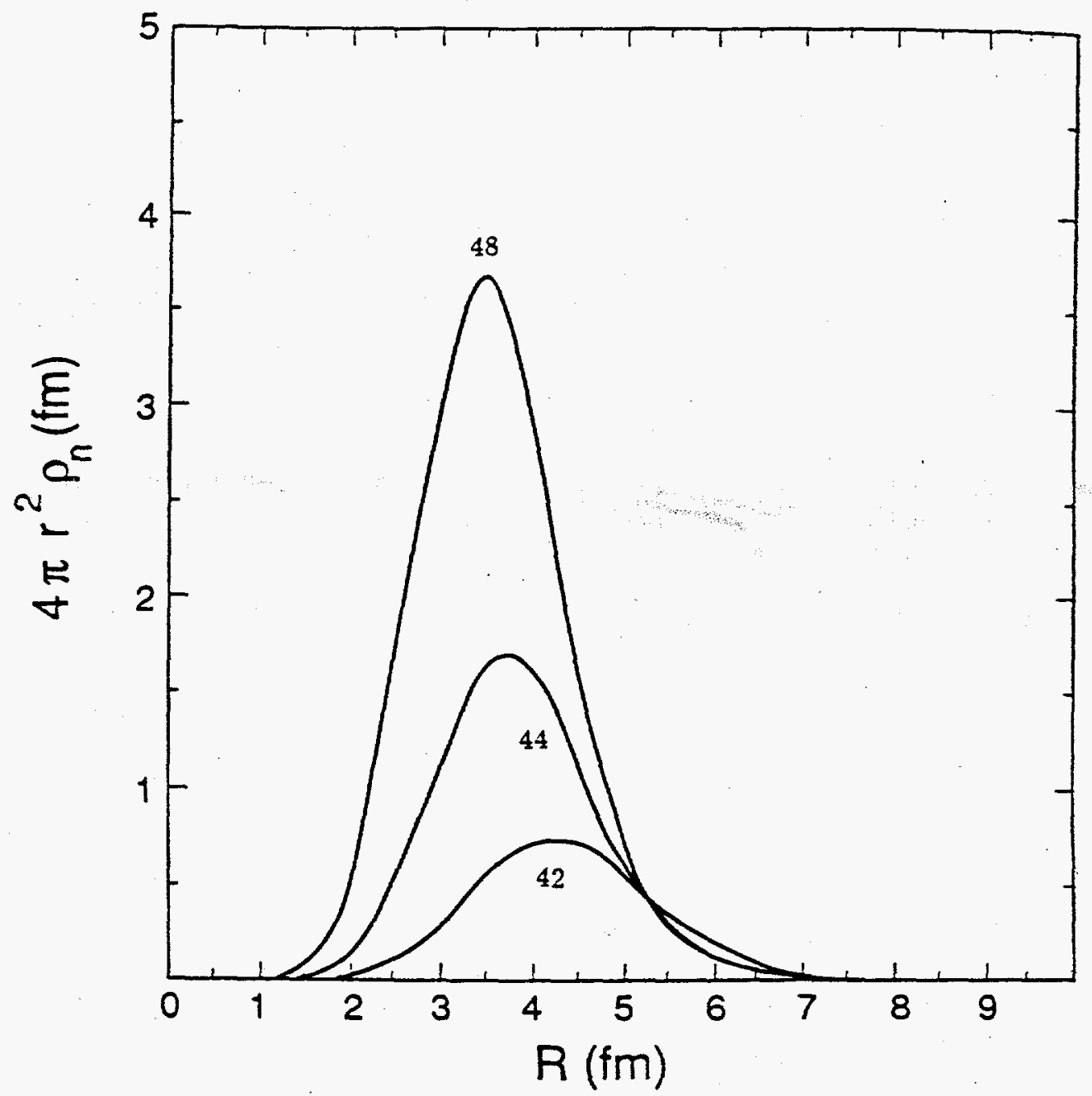

Figure 3.10: Valence neutron densities (multiplied by $r^{2}$ ) inferred in the present work. The shift in the radius toward smaller values with increasing mass number is clearly seen. Most calculations assume that this radius remains constant. 


\subsection{The Hadronic Double Scattering Operator}

In a previously published paper we[28] showed that, due to the simple nuclear structure of the calcium isotopes, the double charge exchange operator can be separated into a long- and short-range part, the range in question being the distance between the two neutrons on which the charge exchanges take place. This separation of ranges takes place, however, in terms of nuclear matrix elements of the fundamental operator. In the recent study [5] we undertook the investigation of the simplest form of the pion double scattering operator, i.e. neglecting the effect of the nucleus which binds the two neutrons on the initial, final and intermediate pion. Even in this very simplified version of the operator certain general features can be discerned. Among these are the cancellation between the non-spin-flip and double-spin-flip parts of the interaction, the separation into 3 distinct zones corresponding to ranges of the operator and the relationship to the classical cross section? - double scattering. Some comparison with the one-pion-exchange part of the nucleon-nucleon interaction is made, in particular the effect of the removal of the $\delta$-function.

\subsection{Pion Scattering and Charge Exchange from Polarized Nuclei}

In the study of the hadronic interaction the spin degree of freedom has always proved to be crucial. For scattering of pions from nuclei, since the pion itself has no spin, the additional handle on the understanding on the interaction must be provided by the polarization of the nucleus itself. Only recently have experiments on polarized nuclei been performed and the results have been poorly reproduced by theories up until now. Peter Siegel and I recently undertook an analysis of the data on elastic scattering, charge exchange and (to a lesser extent) inelastic scattering. The results of this study comparing with data[30, 31, 32] were published in Physical Review [7].

In this first cut we restricted ourselves to reactions on spin $1 / 2$ nuclei (although we have done some work on higher spins). We find for ${ }^{13} \mathrm{C}$ that the data on elastic scattering and charge exchange is well understood with the exception of certain forward angle points (see Figure 3.11). For elastic scattering from ${ }^{15} \mathrm{~N}[32]$ we find an improved agreement with the data over previous calculations[33] but the theoretical asymmetry is still somewhat too large (see Figure 3.12).

Included in the study is a comparison of the optical model approach used by one other theoretical group and the distorted wave impulse approximation that we have 
employed. A short critique of the two methods is given. We also give a discussion of how the relative phases of the amplitudes are related to the underlying potentials to give a physics insight into the polarization asymmetry.

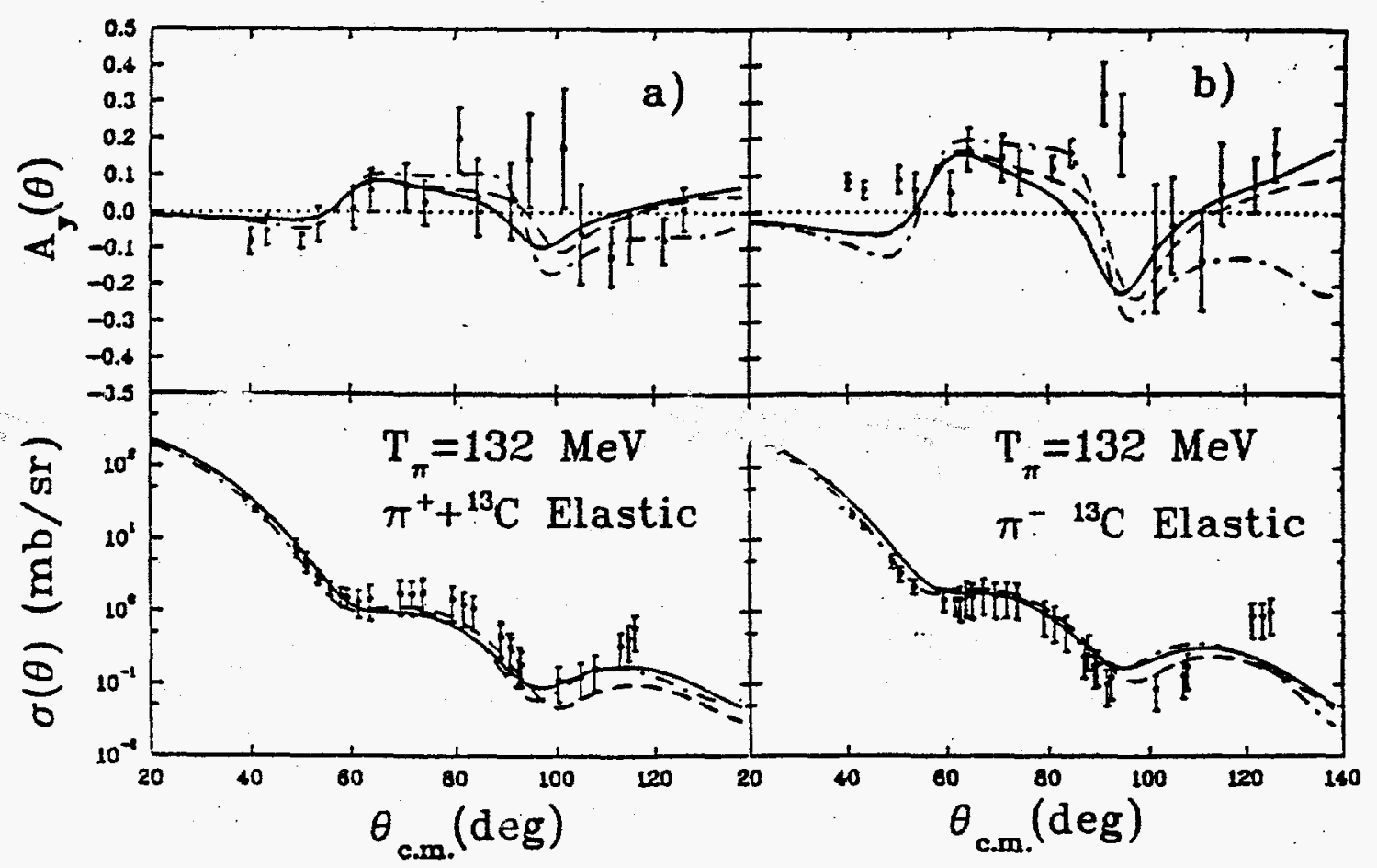

Figure 3.11: Comparison of theory and experiment for asymmetry and differential cross sections at $132 \mathrm{MeV}$ for elastic scattering from ${ }^{13} \mathrm{C}$. The difference in magnitude for the two charges of the pions is clearly seen in both the theory and data. The solid line is the result obtained from distorted waves computed with the pure first-order optical potential and the dashed line is the result obtained by adding an imaginary potential proportional to " $\rho$ " to the optical potential to account for "true" pion absorption. 


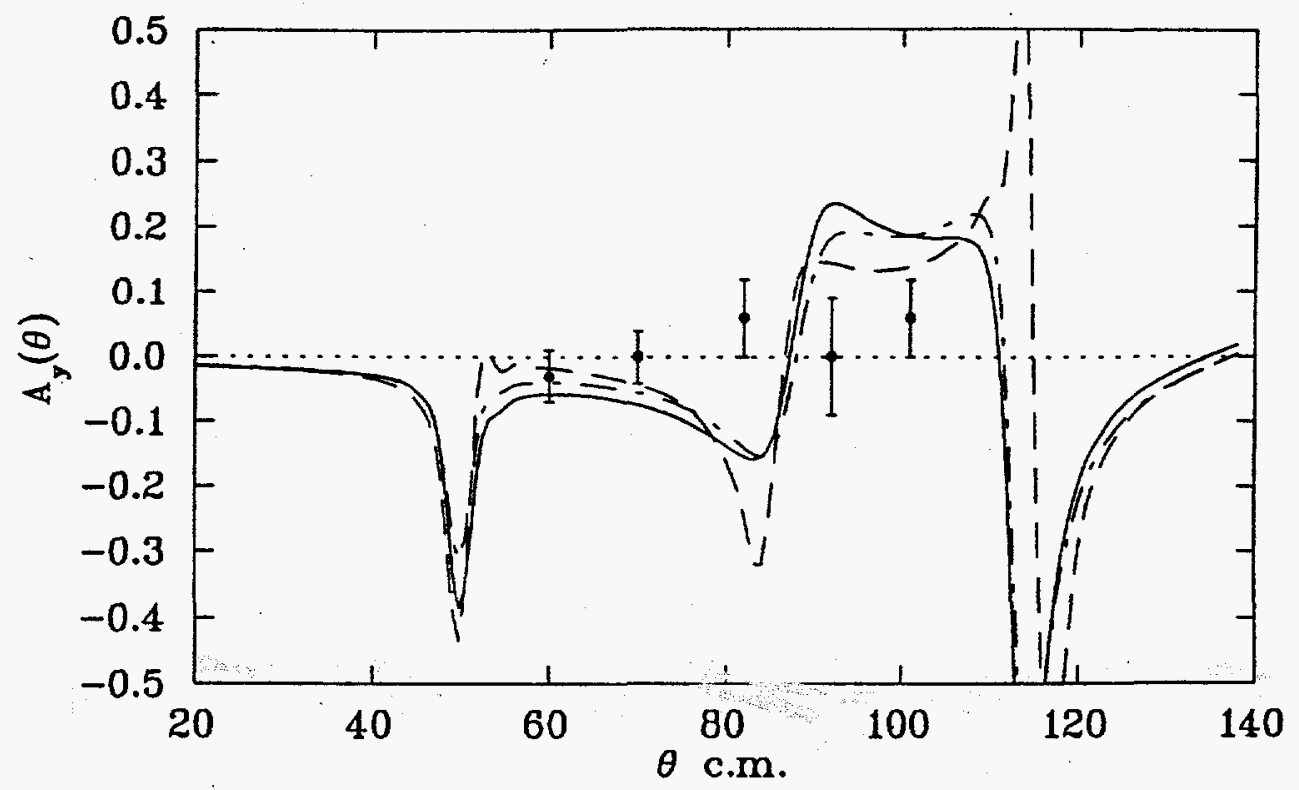

Figure 3.12: Comparison of theory and experiment (at $164 \mathrm{MeV}$ ) for the asymmetry in pion elastic scattering from ${ }^{15} \mathrm{~N}$. Theoretical curves are shown at $150 \mathrm{MeV}$ (dash-dot), $164 \mathrm{MeV}$ (solid) and $170 \mathrm{MeV}$ (dash) to show the change in character of the asymmetry at the resonance (at least in the theory) and to give an idea of the sensitivity to small energy shifts. The agreement is satisfactory, within errors, except for the point at $\theta=82^{\circ}$.

\subsection{Pion Absorption in Nuclei}

While the concept of the equivalence of mass and energy was introduced at the beginning of this century and the conversion between the two is commonplace in modern physics, our understanding of how this transformation actually takes place is minimal. It would seem that it involves a quark-antiquark annihilation in the case of the conversion of the pion mass to pure kinetic energy in a nucleus but how does the reaction take place?

Pion absorption on one nucleon cannot occur in free space due to the conservation of energy and momentum and even in a nuclear medium the missing momentum must be supplied by the Fermi motion. Since the momentum needed is of the order of 500 $\mathrm{MeV} / \mathrm{c}$ the cross section for this one-nucleon process is very small.

In the case of two nucleons the extra momentum can be shared in an equal and opposite manner by the two final nucleons and the absorption on the deuteron is well 
measured and moderately well understood[34]. Since two nucleons are all that are needed to "solve" the momentum problem it was generally assumed that even in a nuclear environment, where there many nucleons around, the absorption takes place on a pair of nucleons.

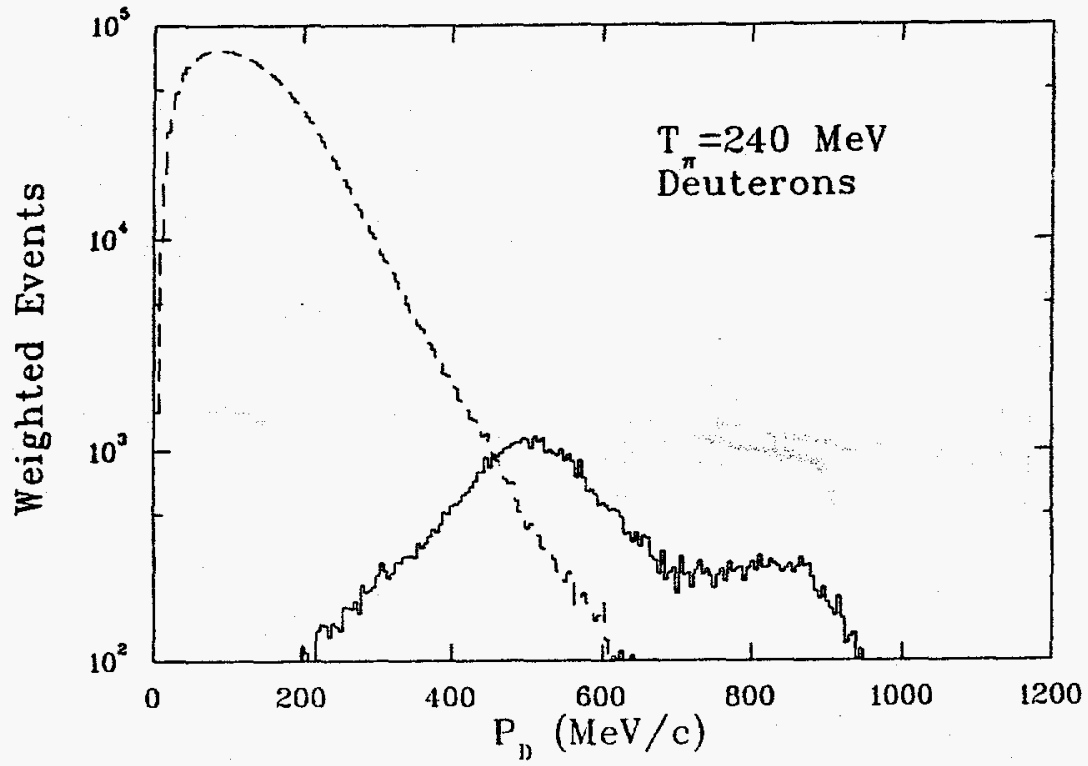

Figure 3.13: Deuteron spectrum from pion absorption on ${ }^{4} \mathrm{He}$. The "spectator" and "pick up" deuterons are both shown.

Attempts to correlate the amount of cross section attributable to two observed nucleons with that of the total measured absorption cross section[35] indicated that a significant fraction was unexplained and apparently had to be attributed to "multinucleon" absorption. Such an observation is potentially very important since it would indicate a many-body mechanism for the conversion of mass to energy in, at least in this case. The difficulty is in trying to firmly establish the existence of this fraction of multi-nucleon absorption (distinguish it from "initial-state"(ISI) and "final-state" (FSI) interactions of the hadrons) and document its properties. Since the initial- and final-state interactions are defined to be on shell (otherwise how can one distinguish many-body from two-body reactions with ISI or FSI) they can be modeled with a classical collision code. There is one caveat, however, in this approach and that is that the wave properties of the pion propagation may need to be treated correctly.

In order to understand these reactions in which several nucleons result from the absorption process the Large Angle Detector and Spectrometer (LADS) was con- 
structed at PSI. The data is now becoming available and I am participating in its analysis.

To this end a classical simulation of the reaction has been constructed which allows rearrangement of clusters. The simulation produces observables which can be compared with the experimental results. One of the more interesting results is a channel in which high energy deuterons result from the pick up of one of the neutrons by one of the final protons resulting from absorption of a $\pi^{+}$. Figure 3.13 show the predicted deuteron spectrum Work is currently in progress (with J.-P. Dedonder) to add quantum effects to the code.

\subsection{Modification of of Nucleon Structure in $\mathrm{Nu}$ - clei}

We suggested a few years ago[36] that a possible way to study the modifications of the structure of the nucleon in a nuclear environment is to compare the total cross section of $\mathrm{K}^{+}$-nucleus scattering with $\mathrm{K}^{+}-\mathrm{d}$ scattering. Since $90 \%$ of the total cross section is given by single scattering this ratio will be sensitive to any changes in the $\mathrm{K}^{+}$-nucleon interaction in the nucleus. In the naive quark model one can show that a simple relationship exists between the confinement range and the s-wave phase shift so that if an increase in size of the nucleon occurs in the nucleus as suggested by Close, Roberts and Ross [37], then the ratio of total cross sections should be larger than that expected from multiple scattering calculations using only standard medium modifications. These measurements have now been made[39, 38] and indeed there does seem to be a renormalization of about the size expected with a definite discrepancy from the fiducial calculation indicated.

The energy dependence seen in the measurements is somewhat different from that predicted originally although those calculations were only meant to be indicative of the size of the effect. Current work centers around the impact of this result on classical nuclear physics.

\subsection{Antiproton Annihilation in Nuclei}

In 1984 D. Strottman and I suggested[40] that the use of energetic antiprotons would provide a useful technique for creating high energy densities within the nucleus. An experiment based on our calculations (and those subsequently done by myself and J. 
Kruk) was proposed by a Rice-BNL collaboration at Brookhaven National Laboratory. The data have been taken and I am participating in its interpretation. As a first stage the charged-particle multiplicities have been extracted and compared with our calculation. They are consistent with the prediction of the IntraNuclear Cascade code with no hadronization time, apparently in contradiction to "conventional wisdom". 


\section{Chapter 4}

\section{Proposed Research}

Nuclear science is at a crossroad. Its future, and indeed the limits of its purview, are ill-defined. The incarnation of this indecision can be expressed in terms of the lack of knowledge of the appropriate (efficient) degrees of freedom to use. We must not confuse this question with that of the fundamental degrees of freedom. It would now appear that QCD is a very strong favorite for that role. However, just as very few would advocate addressing the problems of chemistry with QED, it is probably not the most effective approach in nuclear physics to always return to QCD for each problem. One would not design a bridge with relativistic or quantum mechanics.

The degrees of freedom needed for a given problem depend on the questions asked. The use of nucleonic degrees of freedom (with two-body forces) has achieved a great deal in low-energy nuclear physics. However, much of this advancement has been brought about by the use of symmetries and general theorems and does not depend on the choice of degrees of freedom. A certain number of problems have resisted this first-order theory.

To go beyond this simple first step we enter into the domain of the structure of the nucleons and hence introduce additional degrees of freedom.

Many approaches are currently being tried. One can attempt to construct the nucleus directly from quark and gluon degrees of freedom. This is indeed an ambitious program and would seem to be doomed (at least in the near future) except for some very important qualitative revelations.

The traditional approach to an intermediate set of degrees of freedom has been the exchange of color-singlet mesons. These mesons would then be understood in terms of quark substructure.

One pion exchange has been very well established as the dominant contributor 
for the long-range part of the internucleon potential. It correlates a very significant body of data on nucleon-nucleon interactions. It predicts the static properties of the deuteron[41], gives the correct phase shifts in high partial waves of nucleon-nucleon scattering and predicts the spin-transfer observables in np charge exchange[9]. We have argued recently[9] that pion exchange between quarks in the substructure of the nucleon increases its region of validity to smaller distances and higher energy than previously believed. The extension of one pion exchange to two (and more) pion exchanges is natural. While it is also natural to consider that the two (or more) pions may interact in flight, the extension to the exchange of the resonances in this interaction as particles themselves is less clear.

In fact, this picture of meson exchange has seen some difficulties recently. Neutronproton charge exchange on nuclei[43] and studies of the Drell-Yan[44] process indicate that there is no excess of pions or antiquarks as was expected in some versions of the theory. The $K^{+}$nucleus scattering $[36,38]$ can be simply understood in terms of an enlarged confinement region for the nucleon but heavy meson exchange theories seem to get a variety of results[42]. While a straightforward application of isospin mixing between the $\rho$ and $\omega$ would seem to give a semiquantitative agreement with isospin breaking in the nucleon-nucleon system[17], questions have been raised in trying to understand this process from the point of view of quark substructure[22]. A direct transposition of this theory to the pion-nucleon system gives the wrong sign for the breaking effect[8].

Does our theory of hadronic interaction need a major overhaul or just a minor tune-up? What new parts are needed? The areas I plan to pursue address these questions.

Pion-nucleon Interaction Since the pion plays a central role in the nucleon-nucleon and nuclear system, it is of prime importance that we understand the structure of the interaction. The structure of the nucleon can be expected to be revealed by the composition of its excited states, traditionally formed by pion-nucleon resonances. The new data to be taken at CEBAF will bear on this question, particularly on the matter of qluonic content of the $P_{11}$ resonance (and the nucleon). I plan to continue my studies of the pion-nucleon system and the excited states of the nucleon.

Spin Structure of the Nucleon Through studies of the spin-transfer variables the 
proper degrees of freedom may be indicated. A number of experiments have been done on spin transfer at high energies but there is no successful theory for these observations[45]. Since the attempts have been largely in terms of quarks perhaps hadronic color singlet exchange is still playing a role at a greater energy than previously believed. We have recently found that one pion exchange (corrected for the $\delta$-function) gives a semiquantitative description of these data up to about $2 \mathrm{GeV} / \mathrm{c}$ where a sudden change takes place in the value of $C_{N N}$. An understanding of this transition would very likely tell us a good deal about hadronic interactions. If hadronic coupling remains important at moderately high momentum transfers, the interpretation of the spin EMC effect[46] and the HERMES[47] measurement could well be impacted through vector meson dominance.

Inclusive and Exclusive Nuclear Reactions The ability to model inclusive nuclear reactions is essential to the extraction of any information with high-energy beams. At CEBAF the final states will consist of hadrons propagating through the nucleus. Even for heavy ion reactions at RHIC, where the primary interaction is expected to take place among the fundamental degrees of freedom, the final particles observed will have passed through a condensation phase where "ordinary" hadronic systems are involved. The clustering into deuterons and heavier nuclei can tell us about the thermodynamic properties of the system at "freeze out."

In the search for "color transparency" we must remember that there are cases in which "hadronic transparency" occurs (in inclusive pion scattering [6] and in lowenergy pion elastic scattering[26]). Without a thorough understanding of these phenomena it will be difficult to know when the fundamental degrees of freedom are being probed. It is not clear why a transparency due to composite structures is any less interesting than that due to quarks, for example.

Polarized Nuclei A natural extension of the study of the spin structure of the nucleon and the original EMC effect is to ask if the spin properties of the nucleon are altered in the nucleus. For this purpose scattering from polarized nuclei is a necessary tool. While all probes will be needed, the pion is again important because of its special role in the nucleon-nucleon interaction and its lack of spin. 


\section{Chapter 5}

\section{Personnel}

The Principal Investigator on this grant is ...... $\mathrm{m}$ R. Gibbs.

Since January 1993 one graduate student, $\mathrm{Li} \mathrm{Ai}$, has been participating in the research effort and supported by the grant. Due to the pressure of other commitments on the part of the student for the spring semester $\mathrm{Li} \mathrm{Ai}$ was taken off of the grant and Lowell Cummings was supported instead. 


\section{Bibliography}

\section{Recent and Current Papers}

[1] W. B. Kaufmann and W. R. Gibbs, "Isønin-Symmetry Breaking in the PionNucleon System", Annals of Physics 214, 84(1992).

[2] W. B. Kaufmann, P. B. Siegel and W. R. Gibbs, "Deeply Bound Pionic States via the $\left(\pi^{-}, \mathrm{p}\right)$ Reaction", Phys. Rev. C46, 1474(1992)

[3] J. Langenbrunner, M. K. Jones, D. Dehnhard, C. L. Morris and W. R. Gibbs, "Evidence for Direct Triton Knockout in the ${ }^{4} \mathrm{He}\left(\pi, \pi^{\prime} \mathrm{t}\right) \mathrm{p}$ reaction", Phys. Rev. Lett. 69, 1508(1992)

[4] W. R. Gibbs and J.-P. Dedonder, "Neutron Radii of the Calcium Isotopes", Phys. Rev. C46, 1825(1992)

[5] W. R. Gibbs, M. Elghossain and W. B. Kaufmann, "Pion Double Charge Exchange Operator", Phys. Rev. C48, 1546(1993)

[6] J. D. Zumbro, C. L. Morris, J. A. McGill, S. J. Seestrom, R. M. Whitton, C. M. Riedel-Edwards, A. L. Williams, M. Braunstein, M. Kohler, B. Kriss, S. Hoibraten, R. J. Peterson, J. Ouyang, J. E. Wise, and W. R. Gibbs, "Inclusive scattering of 500-MeV Pions from Carbon", Phys. Rev. Lett. 71, 1796(1993)

[7] P. B. Siegel and W. R. Gibbs, "Pion scattering from spin 1/2 nuclei", Phys. Rev. C48, 1939(1993)

[8] W. R. Gibbs, Li Ai, Mohini Rawool-Sullivan and W. B. Kaufmann, "Isospin breaking in the pion-nucleon interaction", Invited presentation at International Conference on Mesons and Nuclei, Dubna, Russia, May 3-8, 1994 
[9] W. R. Gibbs and B. Loiseau, "Neutron-proton Charge Exchange", Phys. Rev. C50, 2742(1994)

[10] W. R. Gibbs, "Computation in Modern Physics", World Scientific Press, Singapore

ISBN 9810220448

QC20.G48 1994

530.1 '5-dc20

CIP 94-35408

[11] W. R. Gibbs, "New Ideas in Pion Physics", LAMPF Users' Group Meeting, August 1994, Los Alamos New Mexico.

[12] W. R. Gibbs, Li Ai and W. B. Kaufmann, "Isospin Breaking in Low-energy Pion-nucleon Scattering", Phys. Rev. Lett. (In Press)

[13] W. R. Gibbs, "Charge Symmetry Breaking in the Pion-Nucleon System", Invited talk at Pion-Nucleon International Conference, July 7-14, 1995

\section{Additional References}

[14] E. Pedroni, K. Gabathuler, J. J. Domingo, W. Hirt, P. Schwaller, J. Arvieux, C. H. Q.Ingram, P. Gretillat, J. Piffaretti, N. W. Tanner and C. Wilkin, Nucl. Phys. A300, 321(1978).

[15] G. R. Smith, D. R. Gill, D. Ottewell, G. D. Wait, P. Walden, R. R. Johnson, R. Olszewski, R. Rui, M. E. Sevior, R. P. Trelle, J. Brack, J. S. Kraushaar, R. A. Ristinen, H. Chase, E. L. Mathie, V. Pafilis, R. B. Schubank, N. R. Stevenson, A. Rinat and Y. Alexander, Phys. Rev. C38, 240(1988); A. S. Rinat and Y. Alexander, Nucl. Phys. A404, 467(1983)

[16] M. D. Kohler, J. T. Brack, B. Clausen, J. J. Kraushaar, B. J. Kriss, R. A. Ristinen, K. Vaziri, G. R. Smith, D. F. Ottewell, M. E. Sevior, R. P. Trelle and N. R. Stevenson, Phys. Rev. C 44, 15(1991)

[17] S. Coon and R. Barrett, Phys. Rev. C36, 2189 (1987); Sidney A. Coon and Michael D. Scadron, Phys. Rev. C26, 562 (1982). 
[18] J. S. Frank et al., Phys. Rev. D28, 1569(1983); J. T. Brack et al., Phys. Rev. C41, 2202(1990); C38, 2427(1988); C34, 1771(1986); U. Wieder et al., Phys. Rev. Lett. 58, 648(1987); C. Joram, $\pi$ N Newsletter No. 3 (1991); SAID interactive dial-in program; W. Beer et al., Phys. Lett. B261, 16,(1991)

[19] P. Y. Bertin et al. Nucl. Phys. B106, 341(1976);

[20] M. E. Sadler et al., $\pi$ N Newsletter, No. 5, 1992; M. Salomon et al., Nucl. Phys., A414, 493(1984); J. Duclos et al., Phys. Lett. B43, 245(1973); D. H. Fitzgerald et al., Phys. Rev. C34, 619(1985); A. Bagheri Phys. Rev. C38, 885(1988); M. E. Sadler, private communication; SAID interactive dial-in program

[21] Birbriar and Gridnve, Gatchina Preprint TH-24-1994-1966

[22] T. Goldman, J.A. Henderson, and A. W. Thomas, Few Body Systems, 12 (1992) 123; G. Krein, A. W. Thomas and A. G. Williams, Phys. Lett. B317 (1993) 293; J. Piekarewicz and A. G. Williams, Phys. Rev. C47 (1993) 2462; T. Hatsuda, E. M. Henley, T. Meissner and G. Krein, Phys. Rev., C49 (1994) 452; K. Maltman, Phys. Lett. B313 (1993) 203

[23] S. Weinberg, N. Y. Acad. Sci. 38, 185(1977)

[24] T. E. O. Ericson, Phys. Lett. B195, 116(1987)

[25] K. H. McNaughton, D. A. Ambrose, P. Coffey, K. Johnston, P. J. Riley, M. W. McNaughton, K. Koch, I. Supek, N. Tanaka, G. Glass, J. C. Hiebert, L. C. Northcliffe, A. J. Simon, D. J. Mercer, D. L. Adams, H. Spinka, R. H. Jeppesen, G. E. Tripard and H. Woolverton, Phys. Rev. C 46, 47 (1992);

M. W. McNaughton, K. Koch, I. Supek, N. Tanaka, K. H. McNaughton, P. J. Riley, D. A. Ambrose, J. D. Johnson, A. Smith, G. Glass, J. C. Hiebert, L. C. Northcliffe, A. J. Simon, D. L. Adams, R. D. Ransome, D. B. Clayton, H. M. Spinka, R. H. Jeppesen and G. E. Tripard Phys. Rev. C 44, 2267 (1991)

[26] W. R. Gibbs, W. B. Kaufmann and J. P. Dedonder, Phys. Lett. B231, 6 (1989).

[27] R. Bilger, H. A. Clement and M. G. Schepkin, Phys. Rev. Lett. 74, 42(1993)

[28] N. Auerbach, W. R. Gibbs, Joseph N. Ginocchio and W. B. Kaufmann, Phys. Rev C 38, 1277 (1988), N. Auerbach, W. R. Gibbs, and E. Piasetzky, Phys. Rev. Lett. 59, 1076(1987). 
[29] K. G. Boyer, W. J. Braithwaite, W. B. Cottingame S. J. Greene, L. E. Smith, C. F. Moore, C. L. Morris, H. A. Thiessen, G. S. Blanpied, G. R. Burleson, J. F. Davis, J. S. McCarthy, R. C. Minehart, and C. A. Goulding, Phys. Rev. C29, 182(1984); Thesis K. G. Boyer University of Texas, 1983 LA-9974-T

[30] J. J. Görgen, J. R. Comfort, J. R. Tinsley, T. Averett, J. DeKorse, B. Franklin, B. G. Ritchie, G. Kyle, A. Klein, B. Berman, G. Burleson, K. Cranston, J. A. Faucett, J. J. Jarmer, J. N. Knudson, S. Pentilä, N. Tanaka, B. Brinkmöller, D. Dehnhard, Yi-Fen Yen, S. Høibråten, H. Breuer, B. S. Flanders, M. A. Khandaker, D. L. Naples, D. Zhang, M. L. Barlett, G. W. Hoffmann and M. Purcell, Phys. Rev. Lett., 66, 2193(1991)

[31] Yi-Fen Yen, B. Brinkmöller, D. Dehnhard, S. M. Sterbenz, Yi-Ju Yu, Brian Berman, G. R. Burleson, K. Crans . Klein, G. S. Kyle, R. Alarcon, T. Averett, J. R. Comfort, J. J. Görgen, B. C. Ritchie, J. R. Tinsley, M. Barlett, G. W. Hoffmann, K. Johnson, C. F. Moore, M. Purcell, H. Ward, A. Williams, J. A. Faucett, S. J. Greene, J. J. Jarmer, J. A. McGill, C. L. Morris, S. Penttilä, N. Tanaka, H. T. Fortune, E. Insko, R. Ivie, J. M. O'Donnell, D. Smith, M. A. Khandaker, and S. Chakravarti, Phys. Rev. Lett., 66, 1959(1991)

[32] R. Tacik, E. T. Boschitz, R. Maier, S. Ritt, M. Wessler, K. Junker, J. A. Konter, S. Mango, D. Renker, B. Brandt, W. Meyer, W. Thiel, P. Chaumette, J. Deregel, G. Durand, J. Fabre, P. A. Amaudruz, R. R. Johnson, G. R. Smith, P. Weber and R. Mach, Phys. Rev. Lett. 63, 1784(1989)

[33] R. Mach and S. S. Kamalov, Nucl. Phys. A511, 601(1990)

[34] B. Goplen, W. R. Gibbs and E. L. Lomon, Phys. Rev. Lett. 32, 1012 (1974).

[35] D. Ashery, I. Navon, G. Azuelos, H. K. Walter, H. J. Pfeiffer and F. W. Schlepütz, Phys. Rev. C23, 2173(1981)

[36] P. B. Siegel, W. B. Kaufmann and W. R. Gibbs, Phys. Rev. C 31, 2184 (1985).

[37] F. Close, R. G. Roberts and C. G. Ross, Phys. Lett. 129B, 346(1989); F. Close, R. L. Jaffe, R. G. Roberts and C. G. Ross, Phys. Rev. D31, 1004(1985)

[38] R. Weiss, J. Aclander, J. Alster, M. Barakat, S. Bart, R. E. Chrien, R. A. Krauss, K. Johnson, I. Mardor, Y. Mardor, S. May Tal-beck, E. Piasetzky, P. 
H. Pile, R. Sawafta, H. Seyfarth, R. L. Stearns, R. J. Sutter, and A. I. Yavin, Phys. Rev. C49, 2569(1994)

[39] E. Piasetzky, Nuovo Cimento 102A, 281(1989)

[40] D. Strottman and W. R. Gibbs, Phys. Lett. 149B, 288(1984)

[41] T. E. O. Ericson and M. Rosa-Clot, Nucl. Phys. A405, 497(1983); J. L. Friar, B. F. Gibson and G. L. Payne, Phys. Rev. C30, 1084(1984); J. L. Ballot and M. R. Robilotta, Phys. Rev. C45, 986(1992); Phys. Rev. C45, 990(1992); J. L. Ballot, A. M. Eiró and M. R. Robilotta, Phys. Rev. C40, 1459(1989)

[42] J. C. Caillon and J. Labarsouque, Phys. Lett. B295, 21(1992)

[43] Chen X. Y. et al., Phys. Rev. C47, 21v, 9993); Mercer et al. Phys. Rev. Lett. 71,684(1993)

[44] D. M. Alde et al., Phys. Rev. Lett. 64, 2479(1990); ibid 66,133(1991); ibid 66, 2285(1991)

[45] S. J. Brodsky, C. E. Carlson and H. Lipkin, Phys. Rev. D20, 2278(1979); G. Ferrar, S. Gottlieb, D. Sivers and G. Thomas, Phys. Rev. D20, 202(1979)

[46] J. Ashman et al., (EMC), Phys. Lett. 206B, 364(1988); Nucl. Phys. B328, $1(1990)$

[47] HERMES Proposal, K. Coulter et al., 1990 Report DESY/PRC 90-1 